\title{
Antibacterial activity assessment of petroleum ether and methanolic extracts of Achyranthes aspera Linn (Amaranthaceae)
}

\author{
Preeti Mishra \\ TGT in Science, Middle School, Angardiha, West Singhbhum (Jharkhand), India \\ Anita Sha \\ PGT in Biology, KV, Chittaranjan (West Bengal), India \\ Poulami Bhakat \\ Department of Life Science Education (DESM), Regional Institute of Education, Bhubaneswar- \\ 751022 (Odisha), India \\ Sudipta Mondal \\ Department of Life Science Education (DESM), Regional Institute of Education, Bhubaneswar- \\ 751022 (Odisha), India \\ Animesh Kumar Mohapatra* \\ Department of Life Science Education (DESM), Regional Institute of Education, Bhubaneswar- \\ 751022 (Odisha), India \\ *Corresponding author. Email: akmncert@gmail.com
}

\section{How to Cite}

Mishra, P. et al. (2020). Antibacterial activity assessment of petroleum ether and methanolic extracts of Achyranthes aspera Linn (Amaranthaceae). Journal of Applied and Natural Science, 12(3): 354 - 364.https://doi.org/10.31018/jans.v12i3.2319

\begin{abstract}
Achyranthus aspera is a common weed and known for various medicinal properties. The aim of the present study was to evaluate the antibacterial activities of different concentrations of methanolic and petroleum-ether leaf extracts of $A$. aspera against three gram-positive bacteria (Micrococcus luteus, Bacillus subtilis, Streptococcus mitis) and six gram-negative bacteria (Escherichia coli, Pseudomonas aeruginosa, Klebsiella pneumonia, Salmonella typhi, Salmonella paratyphi A (MTCC-3220), Shigella flexneri). The phytochemical screening of the leaf extract of the herb indicated the presence of flavonoides, tannins, saponins, polyphenolic compounds, alkaloids and glycosides. The methanolic extract at the highest concentration of $10 \mathrm{mg} / \mathrm{ml}$ showed prominent antibacterial activity in two gram-negative bacteria, i.e. K. pneumoniae and $E$. coli with $22 \mathrm{~mm}$ zone of inhibition and one gram-positive bacterium i.e. M. luteus with $19 \mathrm{~mm}$ zone of inhibition. The methanolic extract at $0.0781 \mathrm{mg} / \mathrm{ml} \mathrm{con}-$ centration showed least antibacterial activity against all tested bacteria produced a zone of inhibition between 10 to $12 \mathrm{~mm}$ while petroleum ether extract of same concentration had moderate antibacterial activity against $S$. flexneri (15 mm zone of inhibition). It can be concluded that novel compounds like flavonoids, tannins, saponins, alkaloid, and polyphenolic compounds in A. aspera leaves have potent antimicrobial property.
\end{abstract}

Keywords: Achyranthus aspera, Antibacterial activity, Gram-negative, Gram-positive, Phytochemical

\section{INTRODUCTION}

The leading cause of World-wide deaths is due to infectious diseases. Though pharmaceutical industries have produced a wide range of antibiotics, resistance to these drugs by bacteria has increased as they have the genetic ability to acquire resistance. These drugresistant bacteria are more pathogenic with high mortality rate and become a great challenge in the pharmaceutical and healthcare industry (Westh et al., 2004). To overcome these antibiotic-resistant bacteria, researchers are looking for alternative and novel drugs. According to the World Health Organization (WHO), medicinal plants would be the best natural source to obtain a variety of compounds for the treatment of various infectious diseases (Vijayan et al., 2007 ). About $80 \%$ of the world's population relies on traditional medicines which has compounds derived from medicinal plants. In comparison to synthetic antibiotics, plant based drugs cause less or no side effects (Burt, 2004; Shariff et al., 2006; Dubey et al., 2011). Phytotherapy, the treatment of disease by the use of plants is a very old practice when a primitive man out of necessity and by intuition began to use plants to 
overcome his sufferings from injuries and diseases. The plants that possess substances which can be used for therapeutic purposes or can be used for the synthesis of a drug are called medicinal plants (Rishikesh, et al., 2013) In recent years, several studies have been conducted to prove the significance of antimicrobial properties of plant extracts and phytochemicals in therapeutic treatments (Rates, 2001; Gordon and David, 2005; Kumar et al., 2010; Singh et al., 2013). Plants have an extraordinary ability to synthesize compounds, i.e. secondary metabolites like terpenoids, quinines, flavonoids, tannins etc. that are responsible for protecting plants from microorganisms, insects and other natural pests (Selvamohon et al., 2012; Singh et al., 2013; Hasan, 2014). Plants accumulate these secondary metabolites in all cells, but their concentration varies in different parts. The leaf is the part where the highest accumulation of these bioactive compounds takes place. In the recent past, there has been a tremendous increase in the use of these plant products for producing antibacterial drugs in developing as well as developed countries.

World Health Organization (WHO) has made an attempt to identify all medicinal plants used globally and listed more than 20,000 species (Srivastav et al., 2011). Out of these, only a small number has been systematically investigated for their antimicrobial activities (Sharma et al., 2009). One of the plants is Achyranthus aspera which has been reported to contain many phytochemicals (Tiwary et al., 2018) but there is a dearth of studies on the antibacterial action of leaves of this plant.

The investigators observed that the plant $A$. aspera has several medicinal properties. But not much work has been reported so far on the in vitro antimicrobial activities of methanolic and petroleum ether extract of leaves of this weed. Hence, the present study was made to assess the antibacterial activity of the leaves of $A$. aspera so that it can be used for human welfare.

\section{MATERIALS AND METHODS}

Plant material: A. aspera Linn (Amaranthaceae) commonly known as Chirchita in Hindi, an annual stiff, erect herb is found as a weed throughout India. It is about 1-2 meter in height, often with a woody base having an angular or ribbed stem, simple or branched from the base with thick, ovate-elliptic or obovaterounded leaves. The flowers are greenish-white, and seeds are reddish-brown in colour. The plants were collected from the campus of the Regional Institute of Education, Bhubaneswar and taxonomically identified by the Department of Botany, Utkal University, Bhubaneswar, Odisha.

Preparation of plant extract: The fresh leaves of the plant were harvested in the month of August and washed properly with distilled water so as to remove dust and other foreign particles. The leaves were shade dried at room temperature in the laboratory with proper ventilation and ambient temperature. The airdried leaves were further dried in a hot air oven at $40^{\circ} \mathrm{C}$ for 24 hours to remove moisture content. The completely dried leaves were ground into powder using a mechanical grinder. Then the dried powdered material was stored in airtight bottles. 60 gram of pulverized leaves were placed in Soxhlet extraction unit and exhaustively defatted with petroleum ether (60$80^{\circ}$ ) for $40-45$ hours. To confirm whether the extraction is complete or not, the extract from the siphon tube of soxhlet is taken in a watch glass. If no residue remained in the watch glass, the extraction was complete, if not the extraction continued. The extracted plant material was then air-dried, repacked in soxhlet apparatus and then successively and thoroughly extracted with methanol for 40-45 hours. The crude extract obtained was filtered and distilled to evaporate the solvent from the extract. The liquid extract was concentrated separately under vacuum, and the resulting dried extract was preserved in a desiccator until further use.

The dried petroleum ether and methanolic extracts were weighed, and the percentage yield was calculated by the formula mentioned below (Truong et al., 2019):

Percent yield of extract $=$ weight of dried extract X 100/

weight of dried plant material

Eq. 1

Percent yield of petroleum ether extract $=6.334 / 160 \mathrm{X}$

$100=3.96 \% \mathrm{w} / \mathrm{w}$

.Eq. 2

Percent yield of methanolic extract $=6.31 / 160 \times 100=$ $10.19 \% \mathrm{w} / \mathrm{w}$ .Eq. 3

Colour of petroleum ether extract was yellowish-green while the colour of methanolic extract: was greenishblack. The percentage yield of methanolic extract, i.e. $10.19 \% \mathrm{w} / \mathrm{w}$ was more than petroleum ether extract, i.e. $3.96 \%$.

Preliminary phytochemical analysis: Preliminary phytochemical screening was performed by following the methodology of Harborne et al. (1998). To check the presence or absence of primary and secondary metabolites, all the extract were subjected to a battery of chemical tests. The extracts of $A$. aspera were analyzed for the presence of carbohydrates, protein, alkaloids, saponins, tannins, triterpenes, cardiac glycoside, anthraquinones, steroid, coumarin and flavonoids by following standard methods (Williamson et al., 1996; Banso and Ngbede, 2006; Ngbede et al., 2008). The different chemical tests performed for phytochemical screening were: (i) for carbohydrates - Fehling's test and Molisch's test; (ii) for cardiac glycosides - Baljet test and Keller-killiani test (for deoxy sugars); (iii) for steroids and triterpenoids - Raymond's test (for lactose 
ring), Liebermann-burchard test, Hansch's test and Seliwanoff's test; for proteins - Millon's test, Xanthoproteic test and Biuret test; (iv) for alkaloids - Dragendorff's test, Mayer's test, Hager's test and Wagner's test; (v) for glucosides - Keller-killiani test, Baljet test and Legal test; (vi) for saponins - Foam test; (vii) for tanins Gelatin-Lead Acetate test; (viii) for phenolic compounds Ferric chloride test; (ix) for flavonoids - Shinoda test and (x) for terpenoids - Liebermann-burchard test.

\section{Antibacterial activity assay}

Test microorganisms used: The in vitro screening for antibacterial activity was carried out using few selected Gastrointestinal, urinary tract and skin infections causing pathogens which included three gram-positive bacteria (Micrococcus luteus, Bacillus subtilis, Streptococcus mitis) and six gram-negative bacteria (Escherichia coli, Pseudomonas aeruginosa, Klebsiella pneumonia, Salmonella typhi, Salmonella paratyphi A (MTCC-3220), Shigella flexneri). These organisms were procured from Microbial Type Culture Collection (MTCC), Institute of Microbial Technology (IMTECH), Chandigarh. These organisms were identified by standard microbiological methods (Murray et al., 1995). The organisms to be tested were inoculated into sterile nutrient agar. After an incubation period of $24 \mathrm{hr}$ at $37^{\circ} \mathrm{C}$, a loop of inoculums was transferred into $5 \mathrm{ml}$ of nutrient broth and incubated for $2 \mathrm{hr}$ at $37^{\circ} \mathrm{C}$ which served as fresh suspension inoculums.

Determination of antibacterial activity: In the present study, two methods, i.e. (1) Agar well-diffusion method and (2) MIC value determination, were carried out to study the antibacterial activity of $A$. aspera.

Agar well-diffusion method: Antibacterial activities of plant extracts were tested by cup diffusion method on nutrient agar medium (Satish et al., 1999). The culture plates were developed by pouring $20 \mathrm{ml}$ of nutrient agar medium (Hi-Media) into sterile Petri plates. The inoculums suspension was spread uniformly over the agar medium using sterile cotton swabs to get uniform distribution of bacteria. Using a flamed borer, four wells (6 $\mathrm{mm}$ diameter) at the corner were made in the media at a distance of $2-3 \mathrm{~cm}$ from the periphery of the plates and then the wells were labelled. $50 \mu$ l of each plant extract at different concentration were poured aseptically into the well. Standard antibiotic and blank (with the same solvent) were used as a positive and negative control, respectively. Then the plates were incubated for $24 \mathrm{hr}$ at $37^{\circ} \mathrm{C}$ to allow maximum growth of the microorganism. The potency of these extracts was recorded by measuring the diameter of zone of inhibition expressed in millimetre using an antibiotic scale, and the diameter of $\geq 10 \mathrm{~mm}$ was considered as inhibitor zone. Triplicates were performed, and the average value was recorded.

MIC value determination (Two-fold serial dilution):
The minimum inhibitory concentration is defined as the lowest concentration able to inhibit any visible bacterial growth on the culture plates. The Minimum Inhibitory Concentration (MIC) of the petroleum ether and methanolic extracts of $A$. aspera against the test microorganism were determined by using serial dilution technique (Reiner, 1982). The MIC is to determine the lowest concentration of an antibacterial agent that appears to inhibit the growth of the bacteria. The density of the bacterial suspension was standardized by using McFarland standard method (McFarland, 1907). The methanolic extract was dissolved in dimethyl formamide or DMF $(6 \%)$, this was previously tested for antibacterial activity against all test bacteria and found to have no antibacterial activity. The petroleum ether extract was solubilised in a mixture of dimethyl formamide and a surfactant SDS (sodium dodecyl sulphate-2\%). A stock solution of $10 \mathrm{mg} / \mathrm{ml}$ was prepared. Subsequently two-fold serial dilutions were made in concentration range of $5 \mathrm{mg} / \mathrm{ml}$, $0.125 \mathrm{mg} / \mathrm{ml}, 0.3125 \mathrm{mg} / \mathrm{ml}$ and $0.7812 \mathrm{mg} / \mathrm{ml}$. Well with DMF served as negative control and well with antibiotics dissolved in DMF served as a positive control. $50 \mu \mathrm{l}$ of diluted plant extract of different concentrations was added in the respective wells and $50 \mu \mathrm{l}$ of DMF for control. Gentamicin $(0.01 \mathrm{mg} /$ well) and Ciprofloxacin $(0.01 \mathrm{mg} /$ well) served as standard antibiotics against gram-negative bacteria while Ampicillin $(10 \mu \mathrm{g} /$ disc) and Bacitracin (8units/disc) were used as standard antibiotic drug against gram-positive bacteria. The plates were incubated for 24 hours at $37^{\circ} \mathrm{C}$. The MIC values of the extracts were determined by two-fold serial dilution assay.

\section{RESULTS}

Phytochemicals present in petroleum ether and methanolic extract: The preliminary analysis of petroleum ether and methanolic extracts of leaves of $A$. aspera revealed the presence of a number of

Table 1. Phytochemicals present in petroleum ether and methanolic extract of leaves of $A$. aspera.

\begin{tabular}{lcc}
\hline $\begin{array}{l}\text { Phytochemical } \\
\text { compounds }\end{array}$ & $\begin{array}{l}\text { Petroleum } \\
\text { ether extract }\end{array}$ & $\begin{array}{l}\text { Methanolic } \\
\text { extract }\end{array}$ \\
\hline Carbohydrates & - & + \\
Alkaloids & - & + \\
Glycosides & - & + \\
Tannins and polyphe- & + & + \\
nolic compounds & & + \\
Saponins & - & + \\
Flavonoids & + & + \\
Proteins and free ami- & - & + \\
no acids & & + \\
Steroids & + & \\
\hline
\end{tabular}


Table 2. Antibacterial activity of petroleum ether and methanol extracts of $A$. aspera leaves at $10 \mathrm{mg} / \mathrm{ml}$ concentration and standard antibiotics against some human pathogenic bacteria at $0.01 \mathrm{mg} / \mathrm{ml}$ and $0.05 \mathrm{mg} / \mathrm{ml}$. (P- Petroleum ether extract, M- Methanolic extract, G- Gentamicin, Cf- Ciprofloxacin, C- Chloramphenicol, Cfx- Cefixime. NZI- No zone of inhibition, NT- Not tested).

\begin{tabular}{|c|c|c|c|c|c|c|}
\hline \multirow{3}{*}{ Microorganisms (Bacteria) } & \multicolumn{6}{|c|}{ Diameter of Zone of Inhibition (in millimeter) } \\
\hline & \multicolumn{2}{|c|}{$\begin{array}{c}\text { Type of Plant } \\
\text { Extract }(\mathrm{mg} / \mathrm{ml})\end{array}$} & \multicolumn{4}{|c|}{ Standard Drugs (mg/disc) } \\
\hline & $P(10)$ & $M(10)$ & $\mathrm{G}(0.10)$ & Cf (0.01) & $C(0.01)$ & Cfx (0.005) \\
\hline M. luteus (Gram+ve) & $15 \pm 0.08$ & $19 \pm 0.67$ & $25.3 \pm 0.57$ & $35.6 \pm 0.57$ & $22.6 \pm 0.57$ & $18.6 \pm 1.52$ \\
\hline S. mitis (Gram+ve) & $13 \pm 1.65$ & $13 \pm 0.56$ & NT & NT & $\mathrm{NZI}$ & $17 \pm 1.00$ \\
\hline B. subtilis (Gram+ve) & $13 \pm 1.87$ & $17 \pm 0.45$ & NT & NT & $\mathrm{NZI}$ & $\mathrm{NZI}$ \\
\hline S. paratyphi (Gram-ve) & $15 \pm 00$ & $16 \pm 0.45$ & $20.6 \pm 0.57$ & $39 \pm 0.00$ & $\mathrm{NZI}$ & $18.6 \pm 0.57$ \\
\hline E. coli (Gram-ve) & $16 \pm 0.14$ & $22 \pm 1.34$ & $31 \pm 0.00$ & $38 \pm 0.00$ & $21 \pm 0.00$ & $20.3 \pm 0.6$ \\
\hline S. flexneri (Gram-ve) & $22 \pm 0.78$ & $14 \pm 1.05$ & $22 \pm 0.00$ & $35.6 \pm 0.57$ & $17.6 \pm 0.6$ & $14.6 \pm 1.52$ \\
\hline$P$. aeruginosa (Gram-ve) & $12 \pm 0.00$ & $14 \pm 0.15$ & $22.6 \pm 0.57$ & $34 \pm 1.00$ & $\mathrm{NZI}$ & $\mathrm{NZI}$ \\
\hline K. pneumoniae (Gram-ve) & $12 \pm 0.66$ & $22 \pm 0.45$ & $21 \pm 0.00$ & $37 \pm 0.00$ & $20 \pm 0.00$ & $15.6 \pm 0.57$ \\
\hline S. typhi (Gram-ve) & $13 \pm 0.11$ & $16 \pm 0.65$ & $21 \pm 0.00$ & $31 \pm 0.00$ & $\mathrm{NZI}$ & $16.6 \pm 1.52$ \\
\hline
\end{tabular}

Table 3. Antibacterial activity of petroleum ether and methanol extracts of $A$. aspera leaves at $5 \mathrm{mg} / \mathrm{ml}$ concentration and standard antibiotics against some human pathogenic bacteria at $0.01 \mathrm{mg} / \mathrm{ml}$ and $0.05 \mathrm{mg} / \mathrm{ml}$. (P- Petroleum ether extract, M- Methanolic extract, G- Gentamicin, Cf- Ciprofloxacin, C- Chloramphenicol, Cfx- Cefixime. NZI- No zone of inhibition, NT- Not tested).

\begin{tabular}{|c|c|c|c|c|c|c|}
\hline \multirow{3}{*}{$\begin{array}{l}\text { Microorganisms } \\
\text { (bacteria) }\end{array}$} & \multicolumn{6}{|c|}{ Diameter of Zone of Inhibition (in millimetre) } \\
\hline & \multicolumn{2}{|c|}{$\begin{array}{c}\text { Type of Plant } \\
\text { Extract }(\mathrm{mg} / \mathrm{ml})\end{array}$} & \multicolumn{4}{|c|}{ Standard Drugs (mg/disc) } \\
\hline & $P(5)$ & M (5) & G (0.01) & Cf (0.01) & $C(0.01)$ & Cfx (0.05) \\
\hline M. luteus (Gram+ve) & $13 \pm 0.00$ & $17 \pm 0.89$ & $25.3 \pm 0.57$ & $35.6 \pm 0.57$ & $22.6 \pm 0.57$ & $18.6 \pm 1.52$ \\
\hline S. mitis (Gram+ve) & $13 \pm 0.45$ & $12 \pm 1.34$ & NT & NT & $\mathrm{NZI}$ & $17 \pm 1.00$ \\
\hline B. subtilis (Gram+ve) & $12 \pm 0.00$ & $14 \pm 0.34$ & NT & NT & $\mathrm{NZI}$ & $\mathrm{NZI}$ \\
\hline S. paratyphi (Gram-ve) & $14 \pm 0.34$ & $19 \pm 0.65$ & $20.6 \pm 0.57$ & $39 \pm 0.00$ & $\mathrm{NZI}$ & $18.6 \pm 0.57$ \\
\hline E. coli (Gram-ve) & $12 \pm 0.09$ & $18 \pm 0.56$ & $31 \pm 0.00$ & $38 \pm 0.00$ & $21 \pm 0.00$ & $20.3 \pm 0.6$ \\
\hline S. flexneri (Gram-ve) & $18 \pm 0.67$ & $15 \pm 0.56$ & $22 \pm 0.00$ & $35.6 \pm 0.57$ & $17.6 \pm 0.6$ & $14.6 \pm 1.52$ \\
\hline P. aeruginosa (Gram-ve) & $11 \pm 0.45$ & $12 \pm 0.34$ & $22.6 \pm 0.57$ & $34 \pm 1.00$ & $\mathrm{NZI}$ & $\mathrm{NZI}$ \\
\hline K. pneumoniae (Gram-ve) & $12 \pm 0.87$ & $21 \pm 0.67$ & $21 \pm 0.00$ & $37 \pm 0.00$ & $20 \pm 0.00$ & $15.6 \pm 0.57$ \\
\hline S. typhi (Gram-ve) & $12 \pm 0.15$ & $13 \pm 1.87$ & $21 \pm 0.00$ & $31 \pm 0.00$ & $\mathrm{NZI}$ & $16.6 \pm 1.52$ \\
\hline
\end{tabular}

Table 4. Antibacterial activity of petroleum ether and methanol extracts of $A$. aspera leaves at $1.25 \mathrm{mg} / \mathrm{ml}$ concentration and standard antibiotics against some human pathogenic bacteria at $1.25 \mu \mathrm{g} / \mathrm{ml}$. (P- Petroleum ether extract, $M-$ Methanolic extract, G- Gentamicin, Cf- Ciprofloxacin, C- Chloramphenicol, Cfx- Cefixime. NZI- No zone of inhibition, NT- Not tested).

\begin{tabular}{|c|c|c|c|c|c|c|}
\hline \multirow{3}{*}{$\begin{array}{l}\text { Microorganisms } \\
\text { (bacteria) }\end{array}$} & \multicolumn{6}{|c|}{ Diameter of Zone of Inhibition (in millimetre) } \\
\hline & \multicolumn{2}{|c|}{$\begin{array}{c}\text { Type of Plant } \\
\text { Extract (mg/ml) }\end{array}$} & \multicolumn{4}{|c|}{ Standard Drugs (mg/disc) } \\
\hline & $P(1.25)$ & $M(1.25)$ & $\mathbf{G}(0.01)$ & Cf $(0.01)$ & $C(0.01)$ & Cfx (0.05) \\
\hline M. luteus (Gram+ve) & $12 \pm 0.09$ & $15 \pm 0.07$ & $25.3 \pm 0.57$ & $35.6 \pm 0.57$ & $22.6 \pm 0.57$ & $18.6 \pm 1.52$ \\
\hline S. mitis (Gram+ve) & $10 \pm 1.54$ & $11 \pm 0.67$ & NT & NT & $\mathrm{NZI}$ & $17 \pm 1.00$ \\
\hline B. subtilis (Gram+ve) & $11 \pm 0.45$ & $13 \pm 0.24$ & NT & NT & $\mathrm{NZI}$ & $\mathrm{NZI}$ \\
\hline S. paratyphi (Gram-ve) & $12 \pm 0.78$ & $19 \pm 0.45$ & $20.6 \pm 0.57$ & $39 \pm 0.00$ & NZI & $18.6 \pm 0.57$ \\
\hline E. coli (Gram-ve) & $11 \pm 1.07$ & $16 \pm 1.67$ & $31 \pm 0.00$ & $38 \pm 0.00$ & $21 \pm 0.00$ & $20.3 \pm 0.6$ \\
\hline S. flexneri (Gram-ve) & $16 \pm 0.55$ & $15 \pm 1.01$ & $22 \pm 0.00$ & $35.6 \pm 0.57$ & $17.6 \pm 0.6$ & $14.6 \pm 1.52$ \\
\hline P. aeruginosa (Gram-ve) & $10 \pm 0.00$ & $11 \pm 0.00$ & $22.6 \pm 0.57$ & $34 \pm 1.00$ & $\mathrm{NZI}$ & $\mathrm{NZI}$ \\
\hline K. pneumoniae (Gram-ve) & $11 \pm 0.76$ & $17 \pm 0.13$ & $21 \pm 0.00$ & $37 \pm 0.00$ & $20 \pm 0.00$ & $15.6 \pm 0.57$ \\
\hline S. typhi (Gram-ve) & $11 \pm 0.88$ & $12 \pm 0.52$ & $21 \pm 0.00$ & $31 \pm 0.00$ & $\mathrm{NZI}$ & $16.6 \pm 1.52$ \\
\hline
\end{tabular}




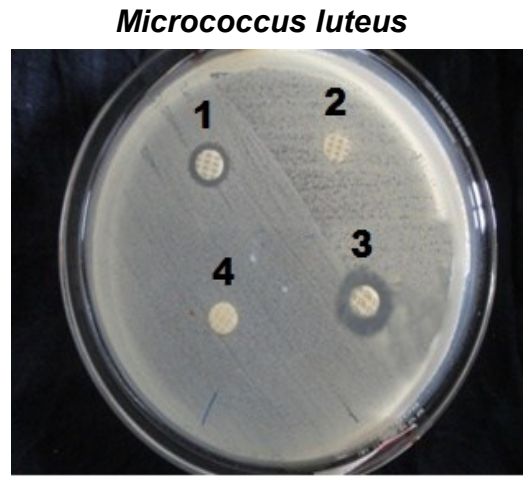

Fig. 1: Antibacterial activity of different standard antibiotics.

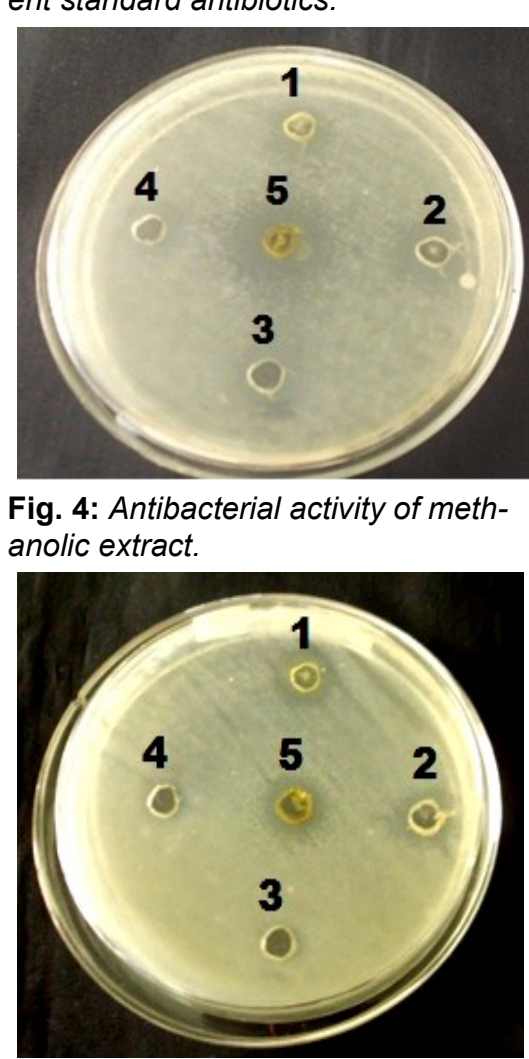

Fig. 7: Antibacterial activity of petroleum ether extract.

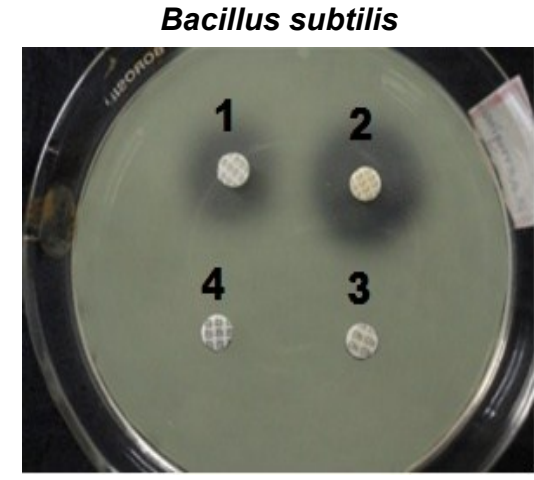

Fig. 2: Antibacterial activity of different standard antibiotics.

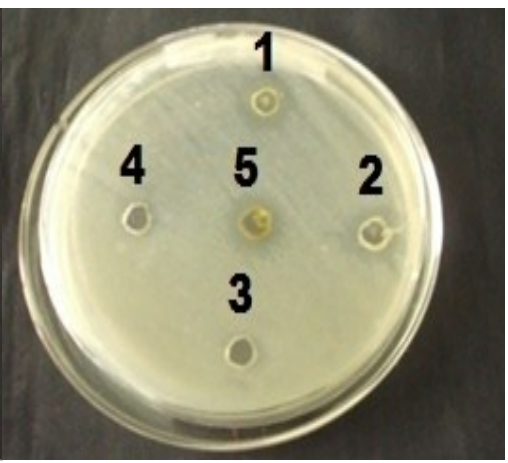

Fig. 5: Antibacterial activity of methanolic extract.

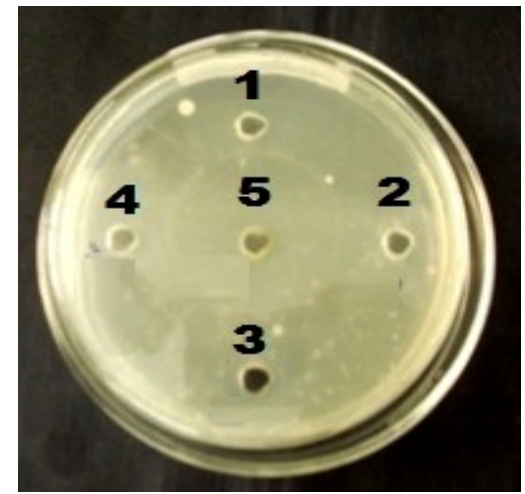

Fig. 8: Antibacterial activity of petroleum ether extract.

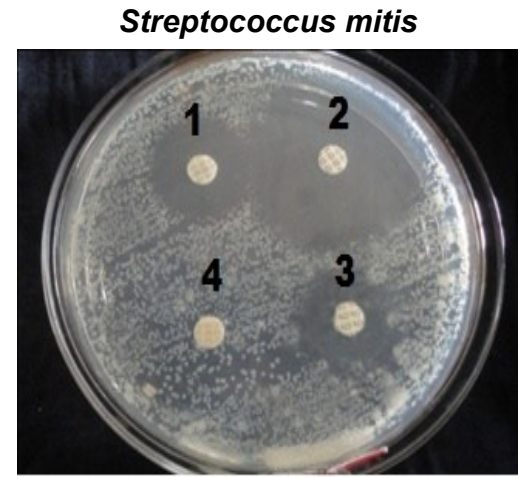

Fig. 3:Antibacterial activity of different standard antibiotics.

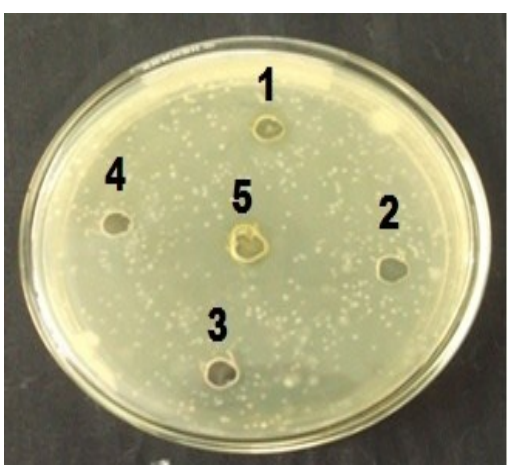

Fig. 6: Antibacterial activity of methanolic extract.

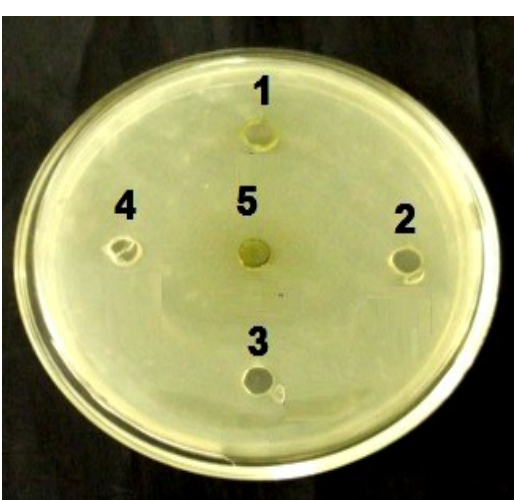

Fig. 9: Antibacterial activity of petroleum ether extract.

Plate 1: Antibacterial activity of standard antibiotics, methanolic and petroleum ether extract against gram positive bacteria; Fig.1 to 3. Showing antibacterial activity of standard antibiotics (1) Ampicillin (10 $\mathrm{gg} / \mathrm{disc}$ ) (2) Bacitracin (8units/disc),

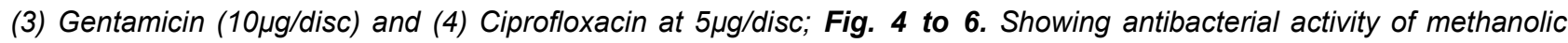
extract. Fig. 7 to 9. Showing antibacterial activity of petroleum ether extract at the concentration of (1) 500 $\mu \mathrm{g} / \mathrm{ml}(2)$ $125 \mu \mathrm{g} / \mathrm{ml}$ (3) $31.25 \mu \mathrm{g} / \mathrm{ml}$ and (4) $7.812 \mu \mathrm{g} / \mathrm{ml}$ (5) $1000 \mu \mathrm{g} / \mathrm{ml}$.

phytochemicals as given in Table 1 .

The petroleum ether and methanolic leaf extracts of $A$. aspera at different concentration showed antibacterial activity against nine different bacteria (M. luteus, $B$. subtilis, S. mitis, E. coli, P. aeruginosa, K. pneumonia, S. typhi, S. paratyphi A (MTCC-3220), S. flexneri).

Antibacterial activity of petroleum ether and methanolic extracts: The methanolic extract at the highest concentration of $10 \mathrm{mg} / \mathrm{ml}$ showed prominent antibacterial activity in two gram-negative bacteria, i.e. K. pneumoniae and $E$. coli with zone of inhibition $22 \mathrm{~mm}$ while $M$. luteus, the gram-positive bacterium showed maximum zone of inhibition i.e. $19 \mathrm{~mm}$. It was moderately active against three gram-negative bacteria i.e. $S$. flexneri, $P$. aeruginosa and $S$. paratyphi with zone of inhibition ranging from $14-16 \mathrm{~mm}$ and one gram-positive bacterium, i.e. B. subtilis showed zone of inhibition of $17 \mathrm{~mm}$. The methanolic extract showed the least antibacterial activity against $S$. mitis with a minimum zone of inhibition of $13 \mathrm{~mm}$. On the other 
Table 5. Antibacterial activity of petroleum ether and methanol extracts of $A$. aspera leaves at $0.3125 \mathrm{mg} / \mathrm{ml}$ concentration and standard antibiotics against some human pathogenic bacteria at $0.3125 \mu \mathrm{g} / \mathrm{ml}$. (P- Petroleum ether extract, M- Methanolic extract, G- Gentamicin, Cf- Ciprofloxacin, C- Chloramphenicol, Cfx- Cefixime. NZI- No zone of inhibition, NT- Not tested).

\begin{tabular}{|c|c|c|c|c|c|c|}
\hline \multirow{3}{*}{ Microorganisms (bacteria) } & \multicolumn{6}{|c|}{ Diameter of Zone of Inhibition (in millimetre) } \\
\hline & \multicolumn{2}{|c|}{ Type of Plant Extract (mg/ml) } & \multicolumn{4}{|c|}{ Standard Drugs (mg/disc) } \\
\hline & $P(0.3125)$ & $M(0.31205)$ & G (0.01) & Cf $(0.01)$ & $C(0.01)$ & Cfx (0.05) \\
\hline M. luteus (Gram+ve) & $13 \pm 0.09$ & $11 \pm 1.67$ & $25.3 \pm 0.57$ & $35.6 \pm 0.57$ & $22.6 \pm 0.57$ & $18.6 \pm 1.52$ \\
\hline S. mitis (Gram+ve) & $10 \pm 0.78$ & $11 \pm 0.56$ & NT & NT & $\mathrm{NZI}$ & $17 \pm 1.00$ \\
\hline B. subtilis (Gram+ve) & $11 \pm 0.23$ & $12 \pm 1.09$ & NT & NT & $\mathrm{NZI}$ & $\mathrm{NZI}$ \\
\hline S. paratyphi (Gram-ve) & $12 \pm 0.78$ & $12 \pm 1.56$ & $20.6 \pm 0.57$ & $39 \pm 0.00$ & $\mathrm{NZI}$ & $18.6 \pm 0.57$ \\
\hline E. coli (Gram-ve) & $10 \pm 1.45$ & $11 \pm 0.34$ & $31 \pm 0.00$ & $38 \pm 0.00$ & $21 \pm 0.00$ & $20.3 \pm 0.6$ \\
\hline S. flexneri (Gram-ve) & $17 \pm 1.00$ & $13 \pm 0.67$ & $22 \pm 0.00$ & $35.6 \pm 0.57$ & $17.6 \pm 0.6$ & $14.6 \pm 1.52$ \\
\hline P. aeruginosa (Gram-ve) & $11 \pm 0.55$ & $10 \pm 1.05$ & $22.6 \pm 0.57$ & $34 \pm 1.00$ & $\mathrm{NZI}$ & $\mathrm{NZI}$ \\
\hline K. pneumoniae (Gram-ve) & $11 \pm 0.56$ & $17 \pm 0.36$ & $21 \pm 0.00$ & $37 \pm 0.00$ & $20 \pm 0.00$ & $15.6 \pm 0.57$ \\
\hline S. typhi (Gram-ve) & $10 \pm 0.88$ & $12 \pm 0.67$ & $21 \pm 0.00$ & $31 \pm 0.00$ & $\mathrm{NZI}$ & $16.6 \pm 1.52$ \\
\hline
\end{tabular}

Table 6. Antibacterial activity of petroleum ether and methanol extracts of $A$. aspera leaves at $0.0781 \mathrm{mg} / \mathrm{ml}$ concentration and standard antibiotics against some human pathogenic bacteria at $0.0781 \mu \mathrm{g} / \mathrm{ml}$. (P- Petroleum ether extract, M- Methanolic extract, G- Gentamicin, Cf- Ciprofloxacin, C- Chloramphenicol, Cfx-Cefixime. NZI- No zone of inhibition, NT- Not tested).

\begin{tabular}{|c|c|c|c|c|c|c|}
\hline \multirow{3}{*}{ Microorganisms (bacteria) } & \multicolumn{6}{|c|}{ Diameter of Zone of Inhibition (in millimetre) } \\
\hline & \multicolumn{2}{|c|}{ Type of Plant Extract (mg/ml) } & \multicolumn{4}{|c|}{ Standard Drugs (mg/disc) } \\
\hline & $P(0.0781)$ & $M(0.0781)$ & $G(0.01)$ & Cf (0.01) & $C(0.01)$ & Cfx (0.05) \\
\hline M. luteus (Gram+ve) & $11 \pm 0.067$ & $10 \pm 1.05$ & $25.3 \pm 0.57$ & $35.6 \pm 0.57$ & $22.6 \pm 0.57$ & $18.6 \pm 1.52$ \\
\hline S. mitis (Gram+ve) & $10 \pm 0.15$ & $12 \pm 0.76$ & NT & NT & $\mathrm{NZI}$ & $17 \pm 1.00$ \\
\hline B. subtilis (Gram+ve) & $10 \pm 1.05$ & $10 \pm 0.54$ & NT & NT & $\mathrm{NZI}$ & NZI \\
\hline S. paratyphi (Gram-ve) & $11 \pm 1.25$ & $12 \pm 0.05$ & $20.6 \pm 0.57$ & $39 \pm 0.00$ & $\mathrm{NZI}$ & $18.6 \pm 0.57$ \\
\hline E. coli (Gram-ve) & $10 \pm 0.34$ & $12 \pm 0.04$ & $31 \pm 0.00$ & $38 \pm 0.00$ & $21 \pm 0.00$ & $20.3 \pm 0.6$ \\
\hline S. flexneri (Gram-ve) & $15 \pm 0.57$ & $10 \pm 0.07$ & $22 \pm 0.00$ & $35.6 \pm 0.57$ & $17.6 \pm 0.6$ & $14.6 \pm 1.52$ \\
\hline$P$. aeruginosa (Gram-ve) & $10 \pm 0.11$ & $10 \pm 0.67$ & $22.6 \pm 0.57$ & $34 \pm 1.00$ & NZI & NZI \\
\hline K. pneumoniae (Gram-ve) & $10 \pm 0.98$ & $12 \pm 0.45$ & $21 \pm 0.00$ & $37 \pm 0.00$ & $20 \pm 0.00$ & $15.6 \pm 0.57$ \\
\hline S. typhi (Gram-ve) & $12 \pm 0.11$ & $12 \pm 1.05$ & $21 \pm 0.00$ & $31 \pm 0.00$ & NZI & $16.6 \pm 1.52$ \\
\hline
\end{tabular}

Table 7. MIC values of Petroleum ether and Methanolic extract of $A$. aspera plant against bacteria tested by serial dilution assay.

\begin{tabular}{llll}
\hline SI. no. & $\begin{array}{l}\text { Microorganisms } \\
\text { (Bacteria) }\end{array}$ & $\begin{array}{l}\text { Petroleum ether extract } \\
\text { MIC }(\mathbf{m g} / \mathbf{m l})\end{array}$ & $\begin{array}{l}\text { Methanolic extract } \\
\text { MIC }(\mathbf{m g} / \mathbf{m l})\end{array}$ \\
\hline 1 & Micrococcus luteus & 0.0781 & 0.3125 \\
2 & Streptococcus mitis & 5 & 0.3125 \\
3 & Bacillus subtilis & 0.3125 & 0.0781 \\
4 & Pseudomonas aeruginosa & 0.3125 & 0.3125 \\
5 & Klebsiella pneumoniae & 0.3125 & 0.0781 \\
6 & Shigella flexneri & 0.0781 & 0.0781 \\
7 & Salmonella typhi & 1.25 & 0.0781 \\
8 & Salmonella paratyphi $A$ & 1.25 & 0.0781 \\
9 & Escherichia coli & 0.3125 & 0.0781 \\
\hline
\end{tabular}

hand, the petroleum ether extract at the concentration of $10 \mathrm{mg} / \mathrm{ml}$ was most effective against one gramnegative bacterium, i.e. S. flexneri while moderately effective against one gram-positive (M. luteus) and two gram-negative (S. paratyphi and $E$. coli) bacteria. The petroleum ether extract was least potent against $P$. aeruginosa and $K$. pneumoniae with minimum zone of inhibition of $12 \mathrm{~mm}$ (Table 2; Plates 1-3).

The methanolic extract at the concentration of $5 \mathrm{mg} / \mathrm{ml}$ amongst gram negative bacteria showed prominent antibacterial activity against E. coli, K. pneumoniae, and $S$. paratyphi. It was moderately active against one gram positive bacterium, i.e. M. luteus, and two gramnegative bacteria, i.e. S. flexneri and $B$. subtilis. The 


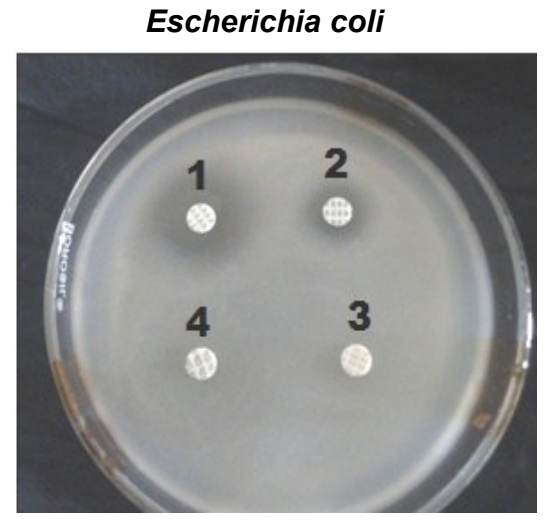

Fig. 10:Antibacterial activity of different standard antibiotics.

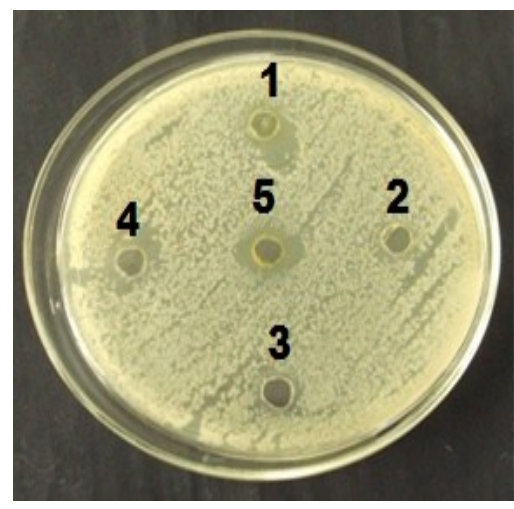

Fig. 13: Antibacterial activity of methanolic extract.

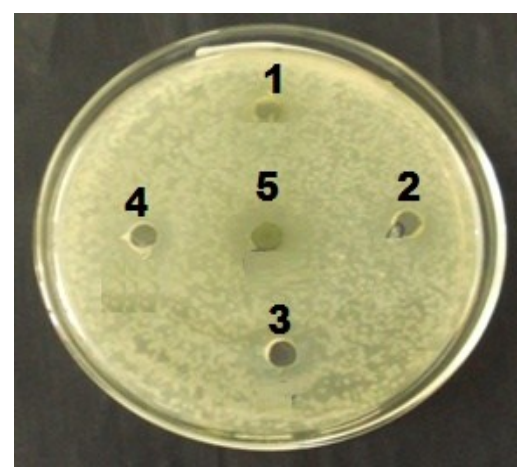

Fig. 16: Antibacterial activity of petroleum ether extract.

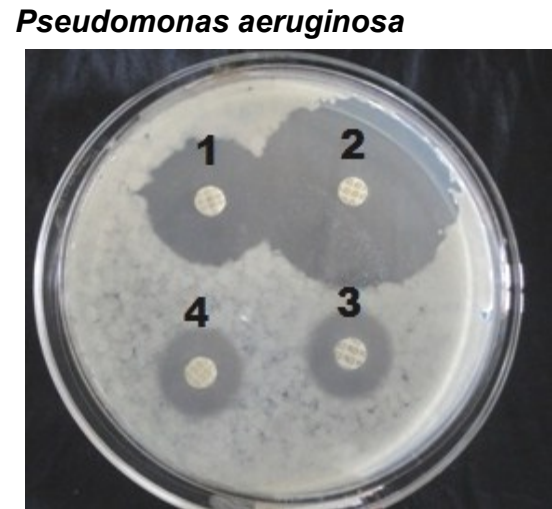

Fig. 11: Antibacterial activity of different standard antibiotics.

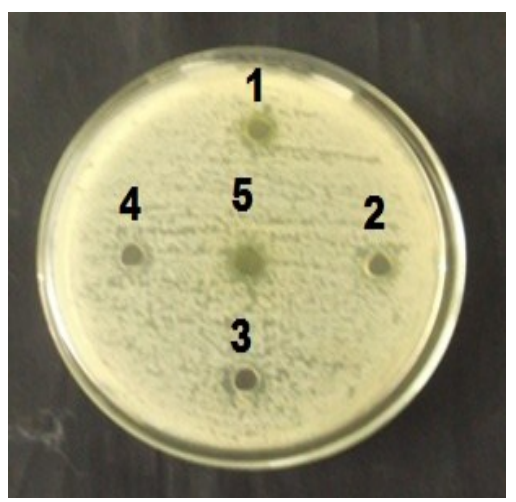

Fig. 14: Antibacterial activity of methanolic extract.

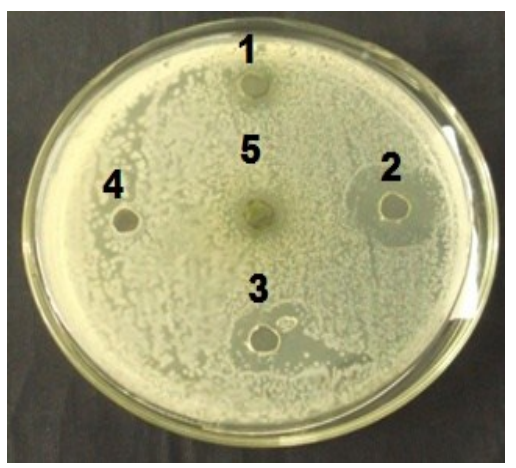

Fig. 17: Antibacterial activity of petroleum ether extract.

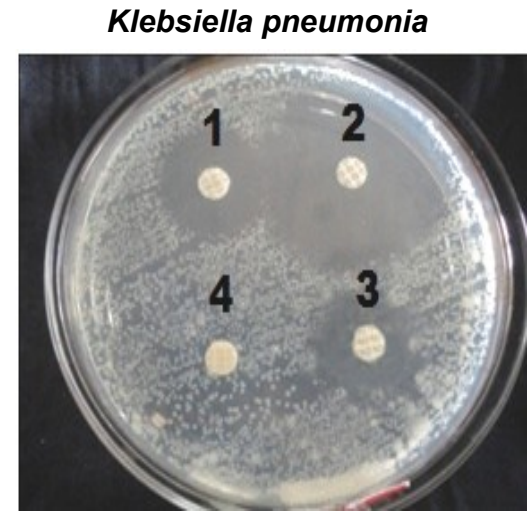

Fig. 12: Antibacterial activity of different standard antibiotics.

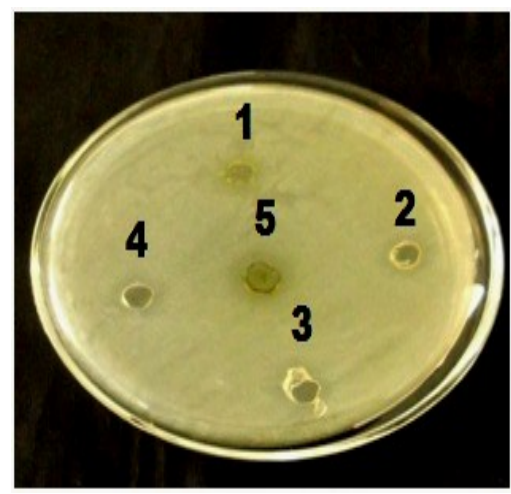

Fig. 15: Antibacterial activity of methanolic extract.

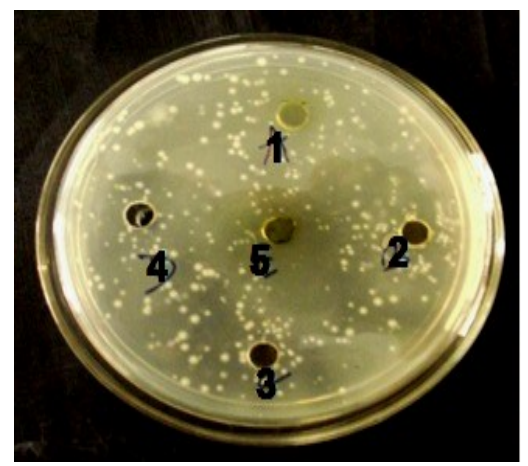

Fig. 18: Antibacterial activity of petroleum ether extract.

Plate 2: Antibacterial activity of standard antibiotics, methanolic and petroleum ether extract against gram negative bac-

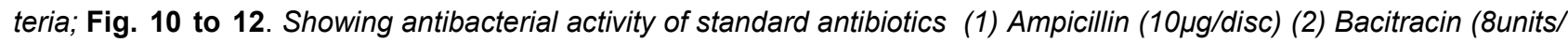

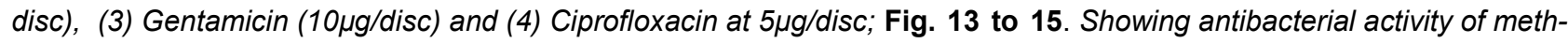
anolic extract and Fig. 16 to 18. Showing antibacterial activity of of petroleum ether extract at the concentration of (1) $500 \mu \mathrm{g} / \mathrm{ml}$ (2) $125 \mu \mathrm{g} / \mathrm{ml}$ (3) $31.25 \mu \mathrm{g} / \mathrm{ml}$ and (4) $7.812 \mu \mathrm{g} / \mathrm{ml}$ (5) $1000 \mu \mathrm{g} / \mathrm{ml}$.

extract was least effective against $S$. mitis and $P$. aeruginosa. On the other hand petroleum ether extract at the diluted concentration show prominent activity against $S$. flexneri but was moderately active against all gram-negative and gram-positive bacteria except for $P$. aeruginosa, $E$. coli and $B$. subtilis with $11-12 \mathrm{~mm}$ as the diameter of zone of inhibition (Table 3; Plates 13).

The methanolic extract at the concentration of $1.25 \mathrm{mg} / \mathrm{ml}$ showed maximum antibacterial activity against S. paratyphi moderate activity against $E$. coli, $S$. flexneri and $K$. pneumococci (Table - 4). The extract was least potent against $S$. mitis and $P$. aeruginosa. The petroleum ether extract was maximum effective against $S$. flexneri while least effective against $S$. mitis and $P$. aeruginosa (Plates- 1 to 3 ).

The methanolic extract at $0.3125 \mathrm{mg} / \mathrm{ml}$ concentration showed prominent antibacterial activity against $K$. pneumonia and showed least effect against $P$. aeruginosa. The petroleum ether extract at same concen- 
Mishra, P. et al. / J. Appl. \& Nat. Sci. 12(3): 354 - 364 (2020)

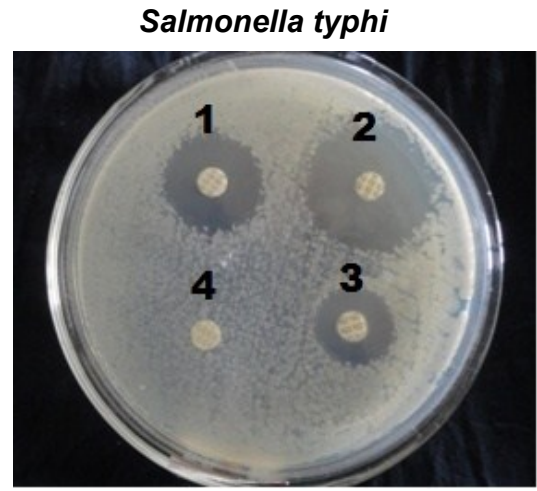

Fig. 19: Antibacterial activity of different standard antibiotics.

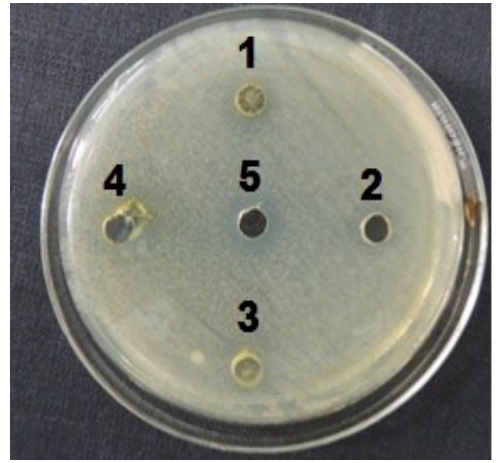

Fig. 22: Antibacterial activity of methanolic extract.

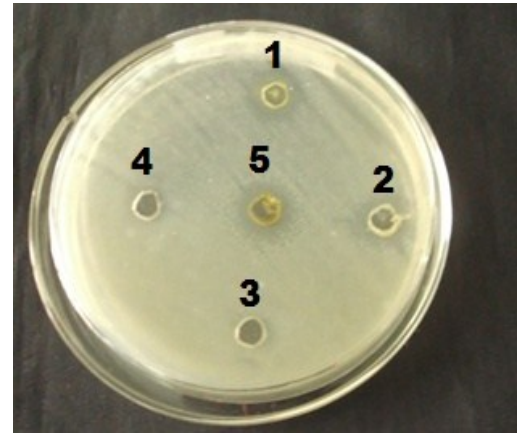

Fig. 25: Antibacterial activity of petroleum ether extract.

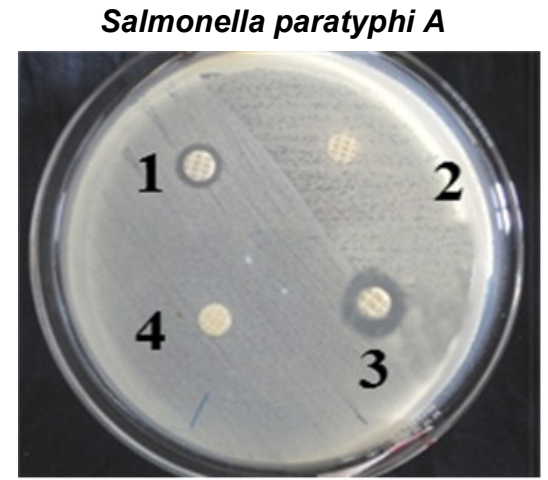

Fig. 20: Antibacterial activity of different standard antibiotics.

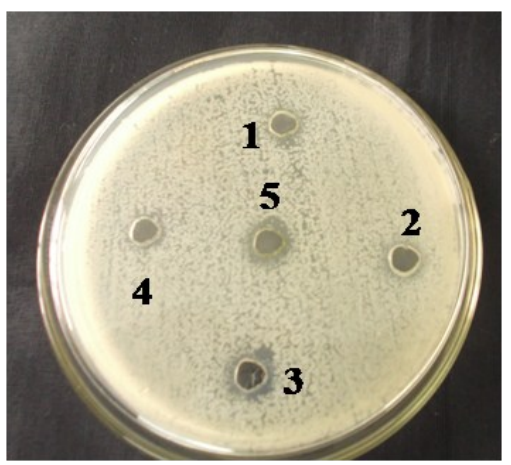

Fig. 23: Antibacterial activity of methanolic extract.

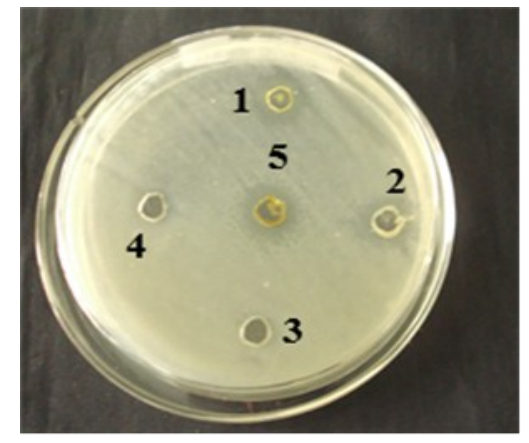

Fig. 26: Antibacterial activity of petroleum ether extract.

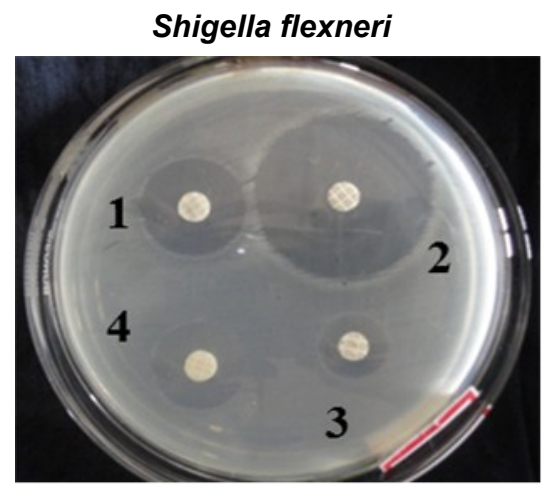

Fig. 21: Antibacterial activity of different standard antibiotics.

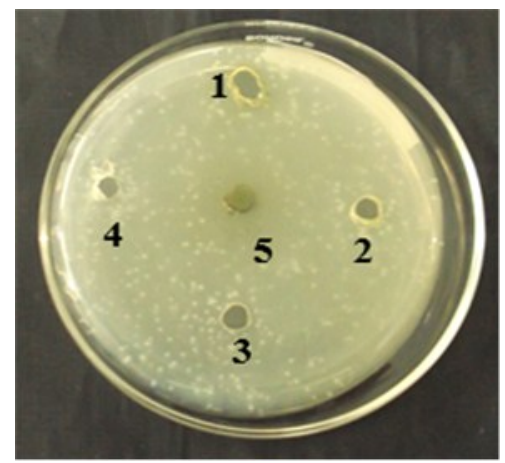

Fig. 24: Antibacterial activity of methanolic extract.

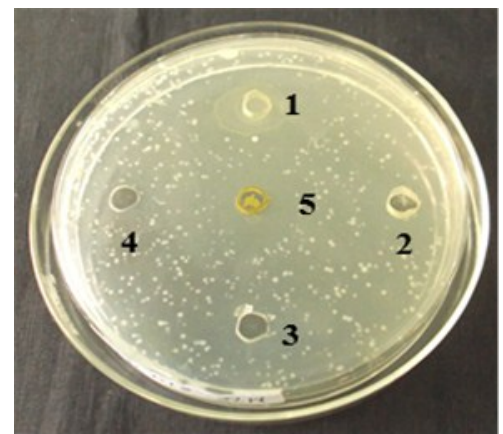

Fig. 27: Antibacterial activity of petroleum ether extract.

Plate 3: Antibacterial activity of standard antibiotics, methanolic and petroleum ether extract against gram negative bac-

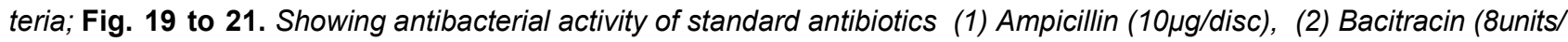

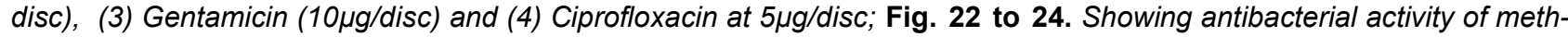
anolic extract and Fig. 25 to 27. Showing antibacterial activity of of petroleum ether extract at the concentration of (1) 500 $\mu \mathrm{g} / \mathrm{ml}$ (2) $125 \mu \mathrm{g} / \mathrm{ml}$ (3) $31.25 \mu \mathrm{g} / \mathrm{ml}$ and (4) $7.812 \mu \mathrm{g} / \mathrm{ml}$ (5) $1000 \mu \mathrm{g} / \mathrm{ml}$.

tration had maximum effect on $S$. flexneri and least effect on $E$. coli and $S$. mitis. The rest bacterial strain showed almost no zone of inhibition indicating its resistance against the extract at $0.3125 \mathrm{mg} / \mathrm{ml}$ concentration (Table 5; Plates 1-3).

The methanolic extract at $0.0781 \mathrm{mg} / \mathrm{ml}$ concentration showed least antibacterial activity against all tested bacteria while petroleum ether extract of same concentration had moderate antibacterial activity against S. flexneri (Table -6$)$. The rest bacterial strain shows almost no zone of inhibition, indicating its resistance against the extract at that concentration (Plates 1-3). Comparative pictorial representations of potency of petroleum ether and methanolic leaf extract of $A$. aspera at different concentrations with that of standard drugs against the tested bacterial strains are shown in Plates- 1 to 3.

Determination of Minimum inhibitory concentration (MIC) against selected bacterial strains: The MIC value of methanolic extract was found to be low 
compared to petroleum-ether extract. The methanolic extract was found to have low MIC values of $0.0781 \mathrm{mg} / \mathrm{ml}$ for most of the bacteria except for $P$. aeruginosa, S. mitis, and $M$. luteus in which the MIC value is $0.3125 \mathrm{mg} / \mathrm{ml}$. With petroleum ether extract $S$. mitis showed a higher MIC value of $5 \mathrm{mg} / \mathrm{ml}$. The lower MIC value is an indication of high effectiveness of the extract, whereas higher MIC indicates the less effectiveness of the extract. The MIC values for different bacteria are given in Table 7 .

\section{DISCUSSION}

The search for novel phytochemicals from natural sources has received much attention and efforts have been put in to identify compounds with the potential to act against antibiotic-resistant bacteria and also to replace synthetic ones. Several researchers have shown that plants are the natural sources of novel compounds like phenols, polyphenols, terpenoids, flavonoids etc that serve as a prototype to develop less toxic and more effective medicines in controlling the growth of microorganisms like Doss et al. (2011) in Medicago sativa; Dubey et al. (2011) in Ziziphus Mauritiana; Mazid et al. (2011) in Rauwolfia canescens; Selvamohon et al. (2012) in Aloe vera, Phyllanthus emblica, Cynodon dactylon; Bagde et al. (2013) in $R$. serpentina; Iqbal et al., (2013) and Rohela et al. (2016) in $R$. tetraphylla. The majority of the active components in $A$. aspera are C-glycosyl flavones based on apigenin and luteolin; Harman alkaloids present in trace amounts along with sucrose and trace amounts of volatile oil (Leung and Froster, 1996; Newall et al., 1996). The major phytoconstituents of this plant are alkaloids, phenols, glycoside, flavonoids, and cyanogenic compounds, maltol, phytosterols, passifloricins, polyketides, and alphapyrones (Dhawan et al., 2004; Tiwari, et al., 2018). In the present study, the methanolic and petroleum ether leaf extract of $A$. aspera revealed the presence of phytochemicals like flavonoids, steroids, Tannins and polyphenolic compounds etc. (Table 1).

Isolation of phytochemical compounds from plant material is largely dependent on the type of solvent used in the extraction procedure. Tiwary et al. (2018), in their study, showed that tannin was present in shoot and root extracts of $A$. aspera but not in the leaf extract. However, the phytochemical analysis of both methanolic and petroleum ether leaf extract of $A$. aspera in the present study clearly showed the presence of tannins. Sravanthi et al. (2013) reported absence of alkaloids and saponins in the alcoholic extracts of leaves of $A$. aspera while the results of the present study and Bajaj et al. (2012) detected the presence of these phytochemicals in methanolic extracts.
The use of several extracts to analyze the efficacy of plants for antimicrobial activity has also been realized by several scientists in many plant species like Adhatoda zeylanica and Trianthema decandra (Geethalakshmi et al., 2010), Argemone mexicana (Rahaman et al., 2011), Tinospora cordifolia, Cassia fistula (Upadhyay, 2011), Ocimum sanctum (Singh et al., 2013) and Carica papaya (Shubam et al., 2019). The traditional practitioners make use of water primarily as a solvent, but the results of the present study showed that organic solvents were certainly much better and powerful. Similar results that methanolic extract is more potent than aqueous extract have been reported by Singh et al. (2013) in O. sanctum. This may be due to the better solubility of the active components in organic solvents and flavonoids are least stable in water which is the primary polyphenolic compound in plants. We found in this study the leaf extract of the $A$. aspera weed by methanol provided more consistent antimicrobial activity compared to those extracted by petroleum ether.

Plant based products have been effectively proven for their utilization as source for antimicrobial compounds. For instance, methanol extracts of $A$. ferox and $W$. somnifera exhibited inhibitory activity against all the strains of $N$. gonorrhea, while only the methanol extract of $W$. somnifera was effective against $C$. albicans (Kambizi and Afolayan, 2008). In the present study, the petroleum-ether and methanolic extracts of leaves of $A$. aspera showed the antimicrobial activity against six gram-negative bacteria ( $E$. coli, $P$. aeruginosa, $K$. Pneumococci. S. typhi, S. paratyphi, S. flexneri) and three gram-positive bacteria (B. subtilis, M. luteus, $S$. mitis (Table 2 - 6, Plates- 1 to 3 ). The methanolic extract showed prominent antibacterial activity against gram negative bacteria, $K$. pneumococci and $E$. coli with a maximum zone of inhibition $(22 \mathrm{~mm}$ ) while it was moderately active against three gram-negative bacteria;, S. paratyphi, S. typhi and $S$. flexineri with zone of inhibition $16 \mathrm{~mm}$ at $10 \mathrm{mg} / \mathrm{ml}$ concentration (Table - 2). The methanolic extract showed least antibacterial activity against $V$. cholera and $P$. aeruginosa with a zone of inhibition of 13-14 mm. Among grampositive bacteria, $M$. luteus showed notable antibacterial activity. On the other hand, petroleum ether extract was most effective against $S$. flexneri while moderately effective against S. paratyphi, E. coli and M. lutes bacteria (Table -2 ). The petroleum ether extract was least potent against $B$. subtilis, $P$. aeruginosa and $K$. pneumococci with a minimum zone of inhibition. The results of the study showed a difference in the effect of different organic solvent extracts for antimicrobial activity and this difference in the activity between different solvent extract is due to the difference between extract compounds in this two extracts. These finding of the 
present study corroborates similar research findings of Abi Beaulah et al. (2011) in A. aspera and Singh et al. (2013) in Ocimum sanctum. They have shown similar antibacterial activity of hexane, chloroform, ethyl acetate and methanol leaf extracts on $S$. aureus, $E$. coli and $P$. mirabilis. The plant may be considered as a biosynthetic laboratory for a multitude of compounds like alkaloid, glycoside, volatile oils, tannins, saponins, flavonoids etc. These compounds are termed as secondary metabolites and are responsible for therapeutic effects.

\section{Conclusion}

The present study concluded that leaf extracts of $A$. aspera contained potential antimicrobial components like flavonoids, saponins, alkaloids and tannins that may be of great use for the development of pharmaceutical industries as a therapy against diabetes (as it possesses hypoglycemic and hypolipidemic activity), cancer and cardiovascular abnormalities (reduces blood pressure and heart rate). The methanol and petroleum ether extracts of $A$. aspera possessed significant inhibitory effect against tested pathogens. The results of the study support the folklore claim along with the development of new antimicrobial drugs from the plant.

\section{ACKNOWLEDGEMENTS}

The authors are greatly indebted to Dr. Sabuj Sahoo, Reader, Department of Pharmaceutical Sciences, Vanivihar, Utkal University, Dr. Sagar Kumar Mishra, Assistant Professor, Department of Pharmaceutical Sciences, Vanivihar, Utkal University and Mr. Sandeep Nayak, Research Scholar, Department of Botany, Vanivihar, Utkal University, Bhubaneswar, Odisha for providing laboratory facilities and for constructive suggestions.

\section{REFERENCES}

1. Abi Beaulah, G., Mohamed, S. A. and aya, S. R. (2011). Antioxidant and antibacterial activity of Achyranthes asera L: An in vitro study. Annals of Biological Research, 2 (5): 662-670.

2. Bagde, S., Khare, M., Patidar, R. K. and Singh, V. (2013). Antimicrobial Properties and Characterization of Phytoconstituents of the Leaf Extracts of Some Medicinal Plants. J. Pharmacognosy Phytochem., 1: 127-132

3. Bajaj, J., Dave, V., Sharma, S., Shukla, A. and Chakole, R. D. (2012). Pharmacognostical and phytochemical studies on Achyranthes aspera. World J. of Pharmacy and Pharmaceutical Sciences, 1 (4): 1316 - 1331.

4. Banso, A. and Ngbede, J. E. (2006). Phytochemical screening and in vitro antifungal properties of Fagara zanthoxyloides. Journal of Food Agriculture and Environment, 4 (3 \& 4): 8-9.

5. Burt, S. (2004). Essential oils: their antibacterial properties and potential applications in foods- A review. Int. J.
Food Microbiol., 94: 223-253.

6. Dhawan, K., Dhawan, S., Sharma, A. (2004). Passiflora: a review update. J. Ethnopharmacol., 94:1-23.

7. Doss, A., V. Parivuguna, M. Vijayasanthi and S. Surendran, (2011).. Antibacterial evaluation and phytochemical analysis of Medicago sativa L. against some microbial pathogens. Ind. J. Sci. Technol., 4: 550-552.

8. Dubey, R., Dubey, K., Sridhar, C., and Jayaveera, K. N. (2011). Human Vaginal Pathogen Inhibition Studies on Aqueous, Methanolic And Saponins Extracts Of Stem Barks Of Ziziphus Mauritiana. Int. J.Pharm. Sci. Res., 2 (3): 659-663.

9. Geethalakshmi, R., Sarada, D. V .L., Marimuthu, P. (2010). Evalution of antimicrobial and antioxidant potentials of Trianthema decandra L. Asian J. of Biotech. 2 (4): 225-231.

10.Gordon, M. C. and David, N. (2005). Biodiversity: A continuing source of novel drug leads. Pure Appl. Chem, 77: 7-24.

11. Harborne, J. B. (1998). Phytochemical methods: A guide to modern techniques of plant analysis. $3^{\text {rd }}$ edition. Chapman \& Hall Pub. London, UK.

12. Hasan S. (2014). Pharmacological and medicinal uses of Achyranthus Aspera. International journal of science, enironmental technology. 3(1):123-129.

13.Iqbal, A.M., A. Firoz, K. Khan and M. Khan, (2013). Ethno -Phyto-Pharmacological Overview on Rauwolfia tetraphylla L. Int. J. Pharm. Phytopharmacol. Res., 2: 247-251.

14.Kambizi, L and Afolayan, A. J. (2008). Extracts from Aloe ferox and Withania somnifera inhibit Candida albicans and Neisseria gonorrhoea. African Journal of Biotechnology, 7 (1):. 012-015,

15.Kumar, G., Karthik, L. and Rao, K.V.B. (2010). Phytochemical composition and in vitro antimicrobial activity of Bauhinia racemosa Lamk (Caesalpiniaceae). International Journal of Pharmaceutical Sciences and Research, 1 (11): 51-58.

16.Leung, A.Y. and Foster, S. (1996) Encyclopedia of Common Natural Ingredients Used in Food, Drugs and Cosmetics. 2nd Edition, John Wiley and Sons, Inc., New York.

17.Mazid, M., T.A. Khan and F. Mohammad, (2011). Role of secondary metabolites in defense mechanisms of plants. Biol. Med., 3: 232-249.

18.McFarland J (1907) The Nephelometer: an instrument for esti-mating the number of bacteria in suspensions used for calculatingthe opsonic index and for vaccines. J Am Med Assoc. 14:1176-1178.

19.Murray, P. R., Baron, E. J., Pfaller, M. A., Tenover, F. C., Yolken, H. R. (1995). Manual of Clinical Microbiology, 6th Ed. ASM Press, Washington DC; 15-18.

20.Newall, C.A., Anderson, L.A. and Phillipson, J.D. (1996). Herbal Medicines. The Pharmaceutical Press, London, pp: 25.

21.Ngbede, J., Yakubu, R. A. and Nyam, D. A. (2008). Phytochemical Screening for Active Compounds in Canarium schweinfurthii ( Atile ) Leaves from Jos North, Plateau State, Nigeria. Medwell Research Journal of Biological Science, 3(9): 1076-1078.

22.Rahman, M. S., Salehin, M. F., Jamal, M. A., Pravin, H. M. and Alam, A. (2011). Antibacterial activity of Arge- 
mone mexicana L. against water borne microbes. Res. J. of Medicinal plant. 5 (5): 621-626.

23.Rates, S. M. K. (2001). Plants as source of drugs. Toxicon., 39: 603-613.

24.Reiner, R. (1982). Antibiotics- An Introduction, F. Hoffman La Roche and Co., Basle Switzerland, p. 70. Research Asia, (2008), 5 (2), 841-844.

25.Rishikesh, M., Rahman, Md. M., Goffar, Md. R., Al Mamun, M. R., Dutta, P. R. and Maruf, Md. A. Al. (2013). Phytochemical and pharmacological investigation of Achyranthes aspera L. Scholar Academic. of Pharmacy, 2 (2): 74-80.

26.Rohela, G. K., Bylla, P., Korra, R. and Reuben, C. (2016). Phytochemical Screening and Antimicrobial Activity of Leaf, Stem, Root and their Callus Extracts in Rauwolfia tetraphylla. Int. J. Agric. Biol., 18 (3): 521 - 528. DOI: 10.17957/IJAB/15.0120

27. Satish. S., Raveesha, K.A. and Janardhana, G.R. (1999). Antibacterial activity of plant extracts on phytopathogenic Xanthomonas campestris pathovars. Letter in Applied Microbiology, 28: 145-147.

28.Selvamohon, T., Ramadas, V. and Kishore, S. S. (2012). Antimicrobial activity of selected medicinal plants against some selected human pathogenic bacteria. Pelagia Research Library, 3 (5): 3374-3381.

29.Shariff, N. S., Sudharshana, M.S., Umesha, S. and Hari Prasad, P. (2006). Antimicrobial activity of Rauwolfia tetraphylla and Physalis minima leaf and callus extracts. Afr. J. Biotechnol., 5: 946-950.

30.Sharma, A., Verma, R. and Ramteke, P. (2009). Antibacterial Activity of Some Medicinal Plants Used by Tribals Against Uti Causing Pathogens. World Applied Sciences Journal, 7 (3): 332-339.

31.Shubham, S., Mishra, R., Gautam, N., Nepal, M., Kashyap, N. and Dutta, K. (2019). "Phytochemical Analysis of Papaya Leaf Extract: Screening Test". EC Dental Science, 18(3): 485- 490.

32.Singh, A. R., Bajaj, B. K., Sekhawat, P. S. and Singh, K. (2013). Phytochemical estimation and antimicrobial activity of aqueous and methanolic extract of Ocimum sanctum
L. J. Nat. Prod. Plant Resour, 3 (1): 51-58.

33.Sravanthi, V., Nagabharathi, M., Manga, P., sowjjanya, B., Usha Rani, B., Bhairagi, P., Sridhar, K. and Umadevi, P. (2013). Invitro antihelmintic activity and preliminary phytochemical analysis of the leaves of Achyranthes aspera. J. Pharm. Biol. Sci., 1 (1): 21-25.

34.Srivastav, S., Singh, P., Mishra, G., Jha, K. K. and Khosa, R. L. (2011). Achyranthes aspera - An important medicinal plant: A review. J. Nat. Prod. Plant Resour., 1 (1): 1-14.

35.Tiwari, P., Gond. P. and Koshale, S. (2018). Phytochemical analysis of different parts of Achyranthes aspera. Journal of Pharmacognosy and Phytochemistry, SP2: 60-62.

36.Truong, D. H., Nguyen, D. H., Anh Ta, N. T., Bui, A. V., Ha Do, T. and Nguyen, H. C. (2019). Evaluation of the Use of Different Solvents for Phytochemical Constituents, Antioxidants, and In Vitro Anti-Inflammatory Activities of Severinia buxifolia. Hindawi Journal of Food Quality, Volume 2019, Article ID 8178294, 9 pages https:// doi.org/10.1155/2019/817829.

37.Upadhyay RK, Tripathi R, Ahmad S Antimicrobial activity of two Indian medicinal plants Tinospora cordifolia (Family: Menispermaceae) and Cassia fistula (Family: Caesalpinaceae) against human pathogenic bacteria. J. of Pharma. Res., 2011; 4(1):167-170.

38.Vijayan, A., Liju, V. B., John, J. V., Reena, Parthipan, B. and Renuka, C. (2007). Traditional remedies of Kani tribes of Kottoor reserve forest, Agasthyavanam, Thiruvananthapuram, Kerala. Indian Journal of Traditional Knowledge, 6 (4): 589-594.

39. Westh, H., Zinn, C.S., Rosdahl, V.T., Sarisa Study Group (2004): An international multicenter study of antimicrobial consumption and resistance in Staphylococcus aureus isolates from 15 hospitals in 14 countries. Microbial Drug Resistance, 10: 169-176.

40.Williamson, E.M., Okpako, D.J. and Evans, F.J. (1996). Pharmacological Methods in Phytotherapy Research. Vol. 1. Selection, Preparation and Pharmacological Evaluation of Plant Material. John Wiley and Sons Ltd, Chichester, England, pp. 9-13. 